\title{
Relevance of class 1 integrons and extended-spectrum $\beta$-lactamases in drug-resistant Escherichia coli
}

\author{
LI-TAO LIU $^{1 *}$, LI-HONG WAN $^{1 *}$, XIAO-HONG SONG ${ }^{1}$, YAO XIONG ${ }^{1}$, SHAO-JU JIN ${ }^{2}$ and LI-MING ZHOU ${ }^{1,3}$ \\ ${ }^{1}$ Department of Pharmacology, West China School of Preclinical and Forensic Medicine, Sichuan University, Chengdu, \\ Sichuan 610041; ${ }^{2}$ Department of Pharmacology, College of Pharmacy, Ningxia Medical University, Yinchuan 750004; \\ ${ }^{3} 985$ Science and Technology Platform for Innovative Drugs, Sichuan University, Chengdu, Sichuan 610041, P.R. China
}

Received March 27, 2013; Accepted July 29, 2013

DOI: $10.3892 / \mathrm{mmr} .2013 .1626$

\begin{abstract}
Escherichia coli is a common cause of community- and hospital-acquired urinary tract infections, and class 1 integrons are the prior elements of gene transference in the capture and distribution of gene cassettes among clinical gram-negative bacillus. In the present study, the resistance of Escherichia coli to antimicrobial agents was investigated. A total of 97 isolates were found to be susceptible to 16 antimicrobial agents and were detected in the production of extended $\beta$-lactamases (ESBLs), distribution of CTX-M-type $\beta$-lactamases, presence and characterization of class 1 integrons and a variable region of integron-positive isolates. Escherichia coli isolates possessing CTX-M $(31 ; 32 \%)$ were detected in 19 isolates (61.5\%). The presence of ESBLs was associated with resistance to penicillins, third-generation cephalosporins, ciprofloxacin, aminoglycosides and monocyclic $\beta$-lactam antibiotics. Escherichia coli isolates $(69 ; 71.1 \%)$ possessed class 1 integrons associated with resistance to ciprofloxacin and numerous third-generation cephalosporins, penicillins, tobramycin and trimethoprim-sulfamethoxazole. The four gene cassette arrangements were as follows: $d$ frA17-aadA5, aadA1, aacC4-cmlAl and $d f r 2 d$, and 8 carried two disparate class 1 integrons. Five isolates presented class 1 integrons containing no gene cassettes. The distribution of ESBLs and class 1 integrons in Escherichia coli were prevalent with drug resistance in Chengdu. In addition, the resistance range of Escherichia coli isolates that harboured ESBLs and carried class 1 integrons were similar. The current study demon-
\end{abstract}

Correspondence to: Professor Li-Ming Zhou, Department of Pharmacology, 3-17 Renmin South Road, Preclinical and Forensic Medical College, Sichuan University, Chengdu, Sichuan 610041, P.R. China

E-mail: zhou108@163.com

*Contributed equally

Key words: extended spectrum $\beta$-lactamases, Escherichia coli, class 1 integrons, gene cassettes, resistance strated the presence of class 1 integrons and ESBLs, which jointly mediate the resistance of Escherichia coli isolates to a number of antibacterial agents.

\section{Introduction}

Escherichia coli, a commensal bacteria of the gastrointestinal tract in humans and animals, is a common cause of community and hospital-acquired urinary tract infections and varies in its susceptibility to antimicrobials (1-3). At present, misuse of fluoroquinolones and third-generation cephalosporins has led to an increasing number of drug-resistant strains of Escherichia coli in China (4).

Previous studies have shown that $65 \%$ of clinical isolates of Escherichia coli produce extended-spectrum $\beta$-lactamases (ESBLs) in China $(5,6)$. ESBL-producing strains are resistant to $\beta$-lactams, fluoroquinolones and aminoglycosides (7). Cefotaxime (CTX)-M-type enzymes are the most common types of ESBLs (8) and are the predominant ESBLs in Enterobacteriaceae in China, causing hospital- and community-acquired infections (4).

In addition, a number of studies have indicated that integrons have developed a highly efficient mechanism for spreading antibiotic resistance determinants $(9,10)$. Class 1 integrons play a crucial role in the dissemination of antibiotic resistance in Gram-negative bacteria and occur frequently in Escherichia coli by carrying and capturing genes via site-specific recombination catalyzed by specific integrase genes (11-14). Class 1 integrons aid in gene transference in the capture and distribution of gene cassettes among clinical Gram-negative bacillus (15).

To date, the correlation between class 1 integrons and ESBLs of Escherichia coli has not been evaluated. Therefore, the current study focused on analyzing the prevalence of class 1 integrons and CTX-M-type enzymes in clinical isolates of Escherichia coli in Chengdu, China between 2009 and 2011 to determine the correlation between class 1 integrons and ESBLs.

\section{Materials and methods}

Samples. A total of 97 non-duplicated clinical Escherichia coli isolates were collected from the sputum of patients from the 
Table I. Primer sequences and PCR.

\begin{tabular}{|c|c|c|c|c|}
\hline Primers & Primer sequence $\left(5^{\prime}-3^{\prime}\right)$ & PCR conditions & Reference & Expected size, bp \\
\hline CTX-M-F & TGTTGTTAGGAAGTGTGCCGC & 1 cycle of $3 \mathrm{~min}$ at $94^{\circ} \mathrm{C}$, & & \\
\hline CTX-M-R & TCGTTGGTGGTGCCATAGTC & $\begin{array}{l}35 \text { cycles of } 1 \text { min at } 94^{\circ} \mathrm{C} \text {, } \\
1 \text { min at } 55^{\circ} \mathrm{C} \text { and } 2 \text { min at } 72^{\circ} \mathrm{C} \\
\text { and } 1 \text { cycle of } 7 \text { min at } 72^{\circ} \mathrm{C} \text {. }\end{array}$ & 17 & 687 \\
\hline IntIl-F & GGGTCAAGGATCTGGATTTCG & 1 cycle of $5 \mathrm{~min}$ at $94^{\circ} \mathrm{C}$ & & \\
\hline IntII-R & ACATGGGTGTAAATCATCGTC & $\begin{array}{l}30 \text { cycles of } 30 \mathrm{sec} \text { at } 94^{\circ} \mathrm{C}, \\
1 \mathrm{~min} \text { at } 63^{\circ} \mathrm{C} \text { and } 40 \mathrm{sec} \text { at } 72^{\circ} \mathrm{C} \\
\text { and } 1 \text { cycle of } 5 \mathrm{~min} \text { at } 72^{\circ} \mathrm{C} \text {. }\end{array}$ & 18 & 484 \\
\hline $5^{\prime} \mathrm{CS}$ & GGCATCCAAGCAGCAAGC & 1 cycle of $3 \min$ at $94^{\circ} \mathrm{C}$ & & \\
\hline $3^{\prime} \mathrm{CS}$ & AAGCAGACTTGACCTGAT & $\begin{array}{l}35 \text { cycles of } 30 \mathrm{sec} \text { at } 94^{\circ} \mathrm{C}, \\
30 \mathrm{sec} \text { at } 55^{\circ} \mathrm{C} \text { and } 1 \mathrm{~min} \text { at } 72^{\circ} \mathrm{C} \\
\text { and } 1 \text { cycle of } 5 \min \text { at } 72^{\circ} \mathrm{C} \text {. }\end{array}$ & 19 & - \\
\hline
\end{tabular}

CTX, cefotaxime; CS, conserved segments; PCR, polymerase chain reaction.

Table II. Comparison of resistance for positive and negative strains of extended spectrum $\beta$-lactamases.

\begin{tabular}{|c|c|c|c|c|c|}
\hline \multirow[b]{2}{*}{ Paper disk of antimicrobial agent } & \multicolumn{2}{|c|}{ ESBLs positive $(\mathrm{n}=31)$} & \multicolumn{2}{|c|}{ ESBLs negative $(\mathrm{n}=66)$} & \multirow[b]{2}{*}{ P-value } \\
\hline & Resistance, \% & Isolates, $\mathrm{n}$ & Resistance, $\%$ & Isolates, $\mathrm{n}$ & \\
\hline CTX & 74.2 & 23 & 34.8 & 23 & $0.000^{\mathrm{a}}$ \\
\hline GEN & 87.1 & 27 & 66.7 & 44 & $0.027^{\mathrm{a}}$ \\
\hline ТOB & 54.8 & 17 & 33.3 & 22 & $0.037^{\mathrm{a}}$ \\
\hline CFP & 58.1 & 18 & 30.3 & 20 & $0.009^{\mathrm{a}}$ \\
\hline SXT & 90.3 & 28 & 50.0 & 33 & $0.000^{\mathrm{a}}$ \\
\hline CAZ & 58.1 & 18 & 22.7 & 15 & $0.001^{\mathrm{a}}$ \\
\hline AMP & 96.8 & 30 & 50.0 & 33 & $0.000^{\mathrm{a}}$ \\
\hline FEP & 61.3 & 19 & 21.2 & 14 & $0.000^{\mathrm{a}}$ \\
\hline $\mathrm{TCY}$ & 90.3 & 28 & 78.8 & 52 & 0.133 \\
\hline CIP & 90.3 & 28 & 53.0 & 35 & $0.000^{\mathrm{a}}$ \\
\hline IPM & 6.5 & 2 & 0.0 & 0 & 0.100 \\
\hline PIP & 93.5 & 29 & 72.7 & 48 & $0.014^{\mathrm{a}}$ \\
\hline SAM & 29.0 & 9 & 0.0 & 0 & $0.000^{\mathrm{a}}$ \\
\hline $\mathrm{TZP}$ & 19.4 & 6 & 3.2 & 1 & $0.004^{\mathrm{a}}$ \\
\hline CRO & 67.7 & 21 & 31.8 & 21 & $0.001^{\mathrm{a}}$ \\
\hline ATM & 51.6 & 16 & 25.8 & 17 & $0.012^{\mathrm{a}}$ \\
\hline
\end{tabular}

${ }^{a} \mathrm{P}<0.05$ vs. negative. ESBLs, extended spectrum $\beta$-lactamases; CTX, cefotaxime; GEN, gentamicin; TOB, tobramycin; CFP, cefoperazone; SXT, trimethoprim-sulfamethoxazole; CAZ, ceftazidine; AMP, ampicillin; FEP, cefepime; TCY, tetracycline, CIP, ciprofloxacin; IPM, imipenem; PIP, piperacillin; SAM, ampicillin-sulbactam; TZP, piperacillin-tazobactam; CRO, ceftriaxone; ATM, aztreonam.

Chengdu No. 7 People's Hospital (Sichuan, China) between 2009 and 2011 and were identified using the Microscan WalkAWay-40 (Siemens, Erlangen, Germany). Written informed consent was obtained from the patients.

Isolate susceptibility. Isolate susceptibility was determined by the disc diffusion technique on Mueller-Hinton agar plates (Oxoid Ltd., Basingtoke, Hampshire, UK) in accordance with CLSI guidelines (16). The following reagents were used: $10 \mu \mathrm{g}$ ampicillin (AMP), $100 \mu \mathrm{g}$ piperacillin (PIP), $30 \mu \mathrm{g}$ ceftazidime (CAZ), $30 \mu \mathrm{g}$ cefepime (FEP), $30 \mu \mathrm{g}$ ceftriaxone (CRO), $35 \mu \mathrm{g}$ aztreonam (ATM), $5 \mu \mathrm{g}$ ciprofloxacin (CIP), $30 \mu \mathrm{g}$ tetracycline (TCY), 10/10 $\mu \mathrm{g}$ ampicillin-sulbactam (SAM), 100/10 $\mu \mathrm{g}$ piperacillin-tazobactam (TZP), $30 \mu \mathrm{g}$ cefotaxime (CTX), $75 \mu \mathrm{g}$ cefoperazone (CFP), $10 \mu \mathrm{g}$ imipenem (IPM), $10 \mu \mathrm{g}$ tobramycin (TOB), $10 \mu \mathrm{g}$ gentamicin (GEN) and 23.75/1.25 $\mu \mathrm{g}$ trimethoprim-sulfamethoxazole (SXT; all Oxoid Ltd.). Escherichia coli ATCC25922 and Staphylococcus aureus ATCC25923 were used as reference strains for susceptibility testing. 
Table III. Comparison of resistance for positive and negative strains of class 1 integrons.

\begin{tabular}{|c|c|c|c|c|c|}
\hline \multirow[b]{2}{*}{ Antimicrobial agent } & \multicolumn{2}{|c|}{ Integron-positive $(n=69)$} & \multicolumn{2}{|c|}{ Integron-negative $(n=28)$} & \multirow[b]{2}{*}{ P-value } \\
\hline & Resistance, \% & Isolates, $\mathrm{n}$ & Resistance, \% & Isolates, $\mathrm{n}$ & \\
\hline CTX & 55.1 & 38 & 28.6 & 8 & $0.015^{\mathrm{a}}$ \\
\hline GEN & 76.8 & 53 & 64.3 & 18 & 1.156 \\
\hline TOB & 50.7 & 35 & 14.3 & 4 & $0.001^{\mathrm{a}}$ \\
\hline CFP & 50.7 & 35 & 10.7 & 3 & $0.000^{\mathrm{a}}$ \\
\hline SXT & 75.4 & 52 & 32.1 & 9 & $0.000^{\mathrm{a}}$ \\
\hline CAZ & 37.7 & 26 & 25.0 & 7 & 0.169 \\
\hline AMP & 73.9 & 51 & 42.9 & 12 & $0.004^{\mathrm{a}}$ \\
\hline FEP & 39.1 & 27 & 21.4 & 6 & 0.074 \\
\hline TCY & 85.5 & 59 & 75.0 & 21 & 0.173 \\
\hline CIP & 73.9 & 51 & 42.9 & 12 & $0.004^{\mathrm{a}}$ \\
\hline IPM & 14.5 & 1 & 14.5 & 1 & 0.496 \\
\hline PIP & 88.4 & 61 & 57.1 & 16 & $0.001^{\mathrm{a}}$ \\
\hline SAM & 13.0 & 9 & 0.0 & 0 & $0.040^{\mathrm{a}}$ \\
\hline TZP & 7.2 & 5 & 7.1 & 2 & 0.676 \\
\hline CRO & 53.6 & 37 & 17.9 & 5 & $0.001^{\mathrm{a}}$ \\
\hline ATM & 36.2 & 25 & 28.6 & 8 & 0.317 \\
\hline
\end{tabular}

${ }^{a} \mathrm{P}<0.05$ vs. negative. ESBLs, extended spectrum $\beta$-lactamases; CTX, cefotaxime; GEN, gentamicin; TOB, tobramycin; CFP, cefoperazone; SXT, trimethoprim-sulfamethoxazole; CAZ, ceftazidine; AMP, ampicillin; FEP, cefepime; TCY, tetracycline, CIP, ciprofloxacin; IPM, imipenem; PIP, piperacillin; SAM, ampicillin-sulbactam; TZP, piperacillin-tazobactam; CRO, ceftriaxone; ATM, aztreonam.

Table IV. Type and arrangement of gene cassette contained in class 1 integrons.

\begin{tabular}{lcccccc}
\hline & \multicolumn{5}{c}{ Sample } \\
\cline { 2 - 6 } Items & 1 & 2 & 3 & 4 & 5 & 6 \\
\hline Integron numbers & $1 \mathrm{D}$ & $3 \mathrm{D}$ & $5 \mathrm{D}$ & $7 \mathrm{D}$ & $9 \mathrm{D}$ & $3 \mathrm{D}+5 \mathrm{D}$ \\
Strains of the total amount & 1 & 4 & 40 & 7 & 5 & 8 \\
Variable length of integrons, bp & 2327 & 549 & 1593 & 934 & 155 & $549+1593$ \\
Total ratio, $\%$ & 1.5 & 6.15 & 61.5 & 10.8 & 7.7 & 12.3 \\
Containing resistance genes & aacC4-cmlA1 & dfr2d & dfrA17-aadA5 & aadA1 & - & dfr2d -dfrA17-aadA5 \\
\hline
\end{tabular}

A phenotypic confirmatory test was performed with $30 \mu \mathrm{g} \mathrm{CTX}, 30 / 10 \mu \mathrm{g}$ cefotaxime-clavulanic acid, $30 \mu \mathrm{g} \mathrm{CAZ}$ and 30/10 $\mu$ g ceftazidime-clavulanic acid (all Becton-Dickinson, Franklin Lakes, NJ, USA) disks on Mueller-Hinton agar. The results were analyzed as previously described (16). Escherichia coli ATCC 25922, Klebsiella pneumoniae ATCC 700603 and Pseudomonas aeruginosa ATCC 27853 were used as controls.

Primers used to amplify CTX-M genes, intIl and conserved segments are presented in Table I with their corresponding cycling conditions.

Polymerase chain reaction (PCR). Each PCR was carried out in a $25-\mu 1$ volume using 1.5 units Taq DNA polymerase (Promega Corporation, Madison, WI, USA) in the reaction buffer provided, which contained $2.5 \mathrm{mM} \mathrm{MgCl}_{2}, 50 \mu \mathrm{M}$ each deoxynucleoside triphosphate, $0.4 \mu \mathrm{M}$ selected primer and
$2 \mu \mathrm{l}$ DNA template. Each PCR product $(10 \mu \mathrm{l})$ was subjected to electrophoresis on $1.2 \%$ agarose gel.

Amplification was performed by a Tpersonal Thermocycler (Biometra, Göttingen, Germany). PCR products were sequenced using an ABI3730 Sequencer (Applied Biosystems, Foster City, CA, USA) and the sequences were compared with the reported sequences from GenBank.

\section{Results}

Antibiotic resistance rates. Antibiotic resistance rates were as follows: TCY, 82.5\%; PIP, 79.4\%; GEN, 73.2\%; AMP and CIP, $64.9 \%$ each; SXT, 62.9\%; CTX, 47.4\%; CRO, 43.3\%; TOB, 40.2\%; CFP, 39.2\%; CAZ, FEP and ATM 34.0\% each; SAM, 9.3\%; TZP, $7.2 \%$; and IPM, $2.1 \%$.

All isolates, with the exception of 3, were sensitive to FEP. Among them, AMP, TCY, aminoglycoside, fluoroquinolones 


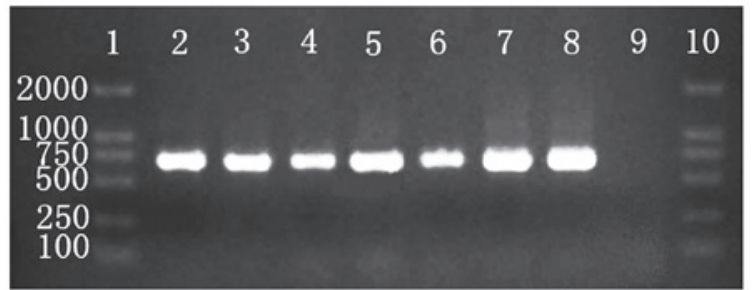

Figure 1. Electrophoregram of CTX-M. Lanes 1, CTX-M (687 bp); 2, positive control; 3-8, experimental group; 9, negative control; and 10, DL2000 marker.

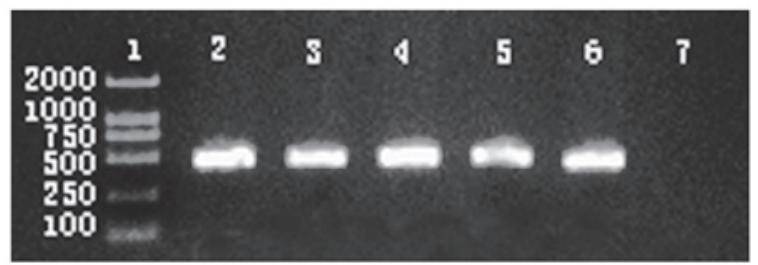

Figure 2. Electrophoregram of intI1 genes. Lanes 1, DL2000 marker; 2, positive group; 3-6, integrase 1; and 7, negative group.

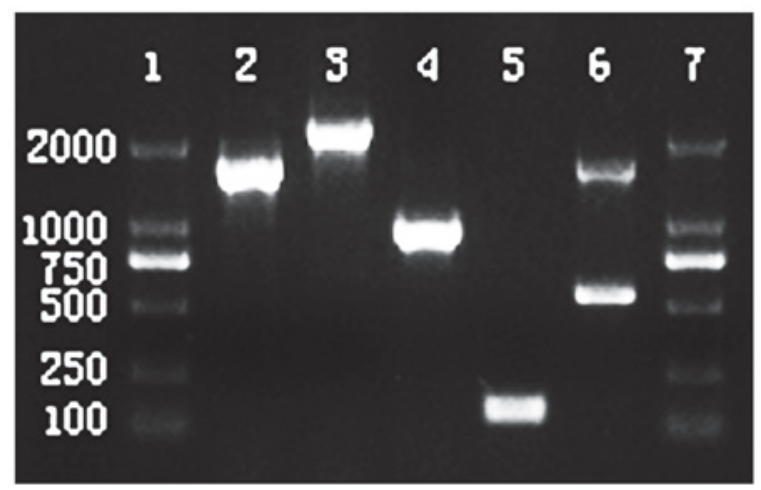

Figure 3. Electrophoregram of variable region in class 1 integron. Lanes 1, DL2000 marker; 2, variable region in class 1 integron; 3, dfr2d (549 bp); 4, aadA1 (934 bp); 5, aacC4+cmlA1 (2,327 bp); 6, dfrA17+aadA5 (1,593 bp); and 7 , no gene cassette arrays (155 bp).

and folic acid metabolic pathway inhibitor resistance was $>60 \%$ and cephalosporin resistance was $\sim 30 \%$. Notably, $2 \%$ of isolates exhibited resistance to IPM.

CTX-M type $\beta$-lactamase production. Based on the phenotypic confirmatory test, 31 isolates $(32 \%)$ were found to be producers of ESBLs. All isolates that tested positive for ESBLs were also multidrug resistant, with a statistically significant difference in resistance against 14 antibacterial drugs between positive and negative isolates $(\mathrm{P}<0.05$; Table II). CTX-M-type $\beta$-lactamase was tested in Escherichia coli isolates (Fig. 1) and detected in 19 isolates $(61.5 \%)$.

Gene cassettes in class 1 integrons. Of the 97 isolates tested, the intIl gene was detected in 69 isolates (71.1\%; Fig. 2) with a statistically significant resistance to 9 antibacterial drugs identified between positive and negative isolates $(\mathrm{P}<0.05$; Table III). Among these class 1 integron gene-positive strains, conserved segments were amplified in 65 isolates $(94.2 \%)$. The amplification products sequenced were $100 \%$ identical to the reported sequence from GenBank. Six conserved segments were detected in the 65 isolates (Fig. 3). Sequence analysis was identical to the following known sequences: $d f r 2 d$ (549 bp; accession no, HQ902143), aadA1 (934 bp; accession no, HQ874618), aacC4-cmlAl (2,327 bp; accession no, HM175867), dfrA17-aadA 5 (1,593 bp; accession no, JN108894) and no gene cassette arrays (155 bp; accession no, FM998811), as presented in Table IV. Four gene cassette arrangements were found in 65/69 intIl-positive isolates (Table IV). The gene cassette arrangements were as follows: aacC4-cmlAl (1.5\%), $d f r 2 d$ (18.45\%), dfrA17-aadA5 (73.8\%), aadAl (10.8\%) and negative control $(7.7 \%)$. The variable region of the following integrons is presented in Fig. 3: $d f r 2 d$ (549 bp), aadAl (934 bp), aacC4+cmlAl (2,327 bp), dfrA17+aadA5 (1,593 bp) and no gene cassette arrays (155 bp). Among them, 8 (12.3\%) of the Escherichia coli isolates carried two integrons and 57 (87.7\%) carried one integron.

\section{Discussion}

As shown in Table II, resistance (\%) was detected in all Escherichia coli isolates of study. Higher resistance to 14 antimicrobial agents was detected in ESBL-positive isolates compared with ESBL-negative isolates $(\mathrm{P}<0.05)$. Resistance to AMP and PIP decreased depending on the inhibition of ESBLs by enzyme inhibitors.

In the current study, a total of 31 isolates (32.0\%) producing ESBLs were identified among the 97 Escherichia coli isolates with the higher prevalence of CTX-M (Fig. 1; 61.3\%, 19/31), consistent with a previous study (20). These observations indicate that the CTX-M group is dominant in Chengdu.

The aacC4, aadAl and aadA5 genes encode resistance to aminoglycosides, $c m l A l$ encodes resistance to chloramphenicols and $d f r 2 d$ and $d f r A 17$ encode resistance to trimethoprim. The gene cassette array $d f r A 17+\operatorname{aadA5}$ is commonly used to detect class 1 integrons $(21,22)$. The prevalence in the present study was lower than that observed previously by Ozgumus et al (23), which showed that all class 1 integron-bearing Escherichia coli contained the aadA5 gene cassette, conferring resistance to streptomycin and spectinomycin. The gene cassette with the lowest detection rate in the present study, aac $4+c m l A 1$, is infrequent in other studies.

Antimicrobial resistance phenotypes were studied in all isolates and the percentages of resistance detected were as follows (Table III; \% integron-positive/\% integron-negative isolates): CTX (55.1/28.6), GEN (76.8/64.3), TOB (50.7/14.3), CFP (50.7/10.7), SXT (75.4/32.1), CAZ (37.7/25.0), AMP (73.9/42.9), FEP (39.1/21.4), TCY (39.1/21.4), CIP (73.9/42.9), IPM (14.5/14.5), PIP (88.4/57.1), SAM (13.0/0), TZP (7.2/7.1), CRO (53.6/17.9) and ATM (36.2/28.6).

In addition, two isolates were resistant to IPM, and a higher percentage of resistance to 9 antimicrobial agents (Table III) was detected among integron-positive isolates compared with integron-negative isolates $(\mathrm{P}<0.05)$. The percentage of multi-resistant strains detected was $62.3 \%(43 / 69)$ among integron-positive isolates and 25.0\% (7/28) among integron-negative isolates $(\mathrm{P}<0.05)$. These observations are in agreement with the hypothesis that class 1 integrons are important in the resistance of Escherichia coli to penicillins, 
third-generation cephalosporins, ciprofloxacin, aminoglycosides and monocyclic $\beta$-lactam antibiotic.

Elevated percentages of resistance were observed in a number of $\beta$-lactam drugs among ESBL-positive isolates compared with ESBLs-negative isolates. The percentages of resistance were as follows (\% ESBL-positive/\% ESBL-negative isolates): CTX (74.2/34.8), GEN (87.1/66.7), TOB (54.8/33.3), CFP (58.1/30.3), SXT (90.3/50.0), CAZ (58.1/22.7), AMP (96.8/50.0), FEP (61.3/21.2), TCY (90.3/78.8), CIP (90.3/53.0), IPM (6.5/0.0), PIP (93.5/72.7), SAM (29.0/0.0), TZP (19.4/3.2), CRO (51.6/25.8) and ATM (51.6/25.8). The percentage of multi-resistant strains detected was $80.6 \%$ (25/31) among ESBL-positive isolates and $37.9 \%(25 / 66)$ among ESBL-negative isolates $(\mathrm{P}<0.05)$. The resistance to third-generation cephalosporins observed was consistent with the existence of ESBLs, as reported by Birgy et al (24).

The resistance profiles of isolates with ESBLs and class 1 integrons are equal. We hypothesize that the presence of the genes resistant to SXT, GEN and TOB in the variable region and ESBLs cause resistance in Escherichia coli isolates to the aforementioned antibacterial drugs. In the current study, 26/31 $(83.9 \%)$ producers of ESBLs were identified to contain class 1 integrons.

The present study indicates that class 1 integrons contributed to the multidrug resistance of Escherichia coli. Class 1 integrons are important for the transfer of resistance genes (25), as the integrons carry antimicrobial-resistant gene cassettes and specific resistance genes correspond to gene cassettes that are detected in clinical isolates of Gram-negative bacteria (26).

The distribution of ESBLs and class 1 integrons in Escherichia coli is prevalent with drug resistance in Chengdu. According to the results of the present study, the presence of class 1 integrons and ESBLs together mediates the resistance of Escherichia coli isolates to the majority of antibacterial agents. Based on our results, we hypothesize that the combined treatment of ESBLs and class 1 integron may offer a new perspective for treating resistant Escherichia coli.

\section{Acknowledgements}

The authors thank the Department of Pharmacology of Preclinical and Forensic Medical College of Sichuan University.

\section{References}

1. Sabharwal ER: Antibiotic susceptibility patterns of uropathogens in obstetric patients. N Am J Med Sci 4: 316-319, 2012.

2. Kang HY, Jeong YS, Oh JY, Tae SH, Choi CH, Moon DC, Lee WK, Lee YC, Seol SY, Cho DT and Lee JC: Characterization of antimicrobial resistance and class 1 integrons found in Escherichia coli isolates from humans and animals in Korea. J Antimicrob Chemother 55: 639-644, 2005

3. Seputiené V, Povilonis J, Ruzauskas M, Pavilonis A and Suziedéliené E: Prevalence of trimethoprim resistance genes in Escherichia coli isolates of human and animal origin in Lithuania. J Med Microbiol 59: 315-322, 2010.

4. Xiao YH, Giske CG, Wei ZQ, Shen P, Heddini A and Li LJ: Epidemiology and characteristics of antimicrobial resistance in China. Drug Resist Updat 14: 236-250, 2011.

5. Jiang HX, Tang D, Liu YH, Zhang XH, Zeng ZL, Xu L and Hawkey PM: Prevalence and characteristics of $\beta$-lactamase and plasmid-mediated quinolone resistance genes in Escherichia coli isolated from farmed fish in China. J Antimicrob Chemother 67: 2350-2253, 2012.
6. Chen YH, Hsueh PR, Badal RE, Hawser SP, Hoban DJ, Bouchillon SK, Ni Y and Paterson DL: Antimicrobial susceptibility profiles of aerobic and facultative Gram-negative bacilli isolated from patients with intra-abdominal infections in the Asia-Pacific region according to currently established susceptibility interpretive criteria. J Infect 62: 280-291, 2011.

7. Wu H, Liu BG, Liu JH, Pan YS, Yuan L and Hu GZ: Phenotypic and molecular characterization of CTX-M-14 extended-spectrum $\beta$-lactamase and plasmid-mediated ACT-like AmpC $\beta$-lactamase produced by Klebsiella pneumoniae isolates from chickens in Henan Province, China. Genet Mol Res 11: 3357-3364, 2012.

8. PatersonDLandBonomoRA:Extended-spectrumbeta-lactamases: a clinical update. Clin Microbiol Rev 18: 657-686, 2005.

9. Han N, Sheng D and Xu H: Role of Escherichia coli strain subgroups, integrons, and integron-associated gene cassettes in dissemination of antimicrobial resistance in aquatic environments of Jinan, China. Water Sci Technol 66: 2385-2392, 2012.

10. Hall RM: Integrons and gene cassettes: hotspots of diversity in bacterial genomes. Ann NY Acad Sci 1267: 71-78, 2012.

11. Woodford N, Carattoli A, Karisik E, Underwood A, Ellington MJ and Livermore DM: Complete nucleotide sequences of plasmids pEK204, pEK499 and pEK516, encoding CTX-M enzymes in three major Escherichia coli lineages from the United Kingdom, all belonging to the international $\mathrm{O} 25: \mathrm{H} 4-\mathrm{ST} 131$ clone. Antimicrob Agents Chemother 53: 4472-4482, 2009.

12. Bennett PM: Plasmid encoded antibiotic resistance: acquisition and transfer of antibiotic resistance genes in bacteria. $\mathrm{Br} \mathrm{J}$ Pharmacol 153 (Suppl 1): S347-S357, 2008.

13. Guérin E, Jové T, Tabesse A, Mazel D and Ploy MC: High-level gene cassette transcription prevents integrase expression in class 1 integrons. J Bacteriol 193: 5675-5682, 2011.

14. van Essen-Zandbergen A, Smith H, Veldman K and Mevius D: Occurrence and characteristics of class 1,2 and 3 integrons in Escherichia coli, Salmonella and Campylobacter spp. in the Netherlands. J Antimicrob Chemother 59: 746-750, 2007.

15. Nardelli M, Scalzo PM, Ramírez MS, Quiroga MP, Cassini MH and Centrón D: Class 1 integrons in environments with different degrees of urbanization. PLoS One7: e39223, 2012.

16. Clinical and Laboratory Standards Institute: Performance standards for antimicrobial susceptibility testing standards: nineteenth informational supplement M100-S19, 2009. Accessed January, 2013.

17. Wu LT, Tsou MF, Wu HJ, Chen HE, Chuang YC and Yu WL: Survey of CTX-M-3 extended-spectrum beta-lactamase (ESBL) among cefotaxime-resistant Serratia marcescens at a medical center in middle Taiwan. Diagn Microbiol Infect Dis 49: 125-129, 2004.

18. Costa D, Poeta P, Sáenz Y, Coelho AC, Matos M, Vinué L, Rodrigues J and Torres C: Prevalence of antimicrobial resistance and resistance genes in faecal Escherichia coli isolates recovered from healthy pets. Vet Microbiol 127: 97-105, 2008.

19. Hsu SC, Chiu TH, Pang JC, Hsuan-Yuan CH, Chang GN and Tsen HY: Characterisation of antimicrobial resistance patterns and class 1 integrons among Escherichia coli and Salmonella enterica serovar Choleraesuis strains isolated from humans and swine in Taiwan. Int J Antimicrob Agents 27: 383-391, 2006.

20. Wu S, Dalsgaard A, Hammerum AM, Porsbo LJ and Jensen LB: Prevalence and characterization of plasmids carrying sulfonamide resistance genes among Escherichia coli from pigs, pig carcasses and human. Acta Vet Scand 52: 47, 2010.

21. Lim KT, Yasin R, Yeo CC, Puthucheary S and Thong KL: Characterization of multidrug resistant ESBL-producing Escherichia coli isolates from hospitals in Malaysia. J Biomed Biotechnol 2009: 165637, 2009.

22. Yang CM, Lin MF, Lin CH, Huang YT, Hsu CT and Liou ML: Characterization of antimicrobial resistance patterns and integrons in human fecal Escherichia coli in Taiwan. Jpn J Infect Dis 62: 177-181, 2009.

23. Ozgumus OB, Celik-Sevim E, Alpay-Karaoglu S, Sandalli C and Sevim A: Molecular characterization of antibiotic resistant Escherichia coli strains isolated from tap and spring waters in a coastal region in Turkey. J Microbiol 45: 379-387, 2007.

24. Birgy A,Cohen R, LevyC,BidetP,Courroux C,Benani M,ThollotF and Bingen E: Community faecal carriage of extended-spectrum beta-lactamase-producing Enterobacteriaceae in french children. BMC Infect Dis 12: 315, 2012.

25. Ramirez MS, Parenteau TR, Centron D and Tolmasky ME: Functional characterization of Tn1331 gene cassettes. J Antimicrob Chemother 62: 669-673, 2008.

26. Bennett PM: Integrons and gene cassettes: a genetic construction kit for bacteria. J Antimicrob Chemother 43: 1-4, 1999. 